\title{
Effects of target luminance and cue validity on the latency of visual detection
}

\author{
HAROLD L. HAWKINS \\ Office of Naval Research, Arlington, Virgina \\ MICHAEL G. SHAFTO \\ NASA, Ames Research Center, Moffett Field, California \\ and \\ KEVIN RICHARDSON \\ Catholic University of America, Washington, D.C.
}

\begin{abstract}
Hughes (1984) has reported that the magnitude of the cue-validity effect in luminance detection is unaffected by target luminance. In three experiments, we explored the possible basis of this counterintuitive finding. The experiments focused on the design of the Hughes study, in which target luminance was treated as a between-blocks variable. Our results reveal that when target luminance is varied randomly within trial blocks, the cue-validity effect grows with declining target luminance. The difference between our findings and those of Hughes is interpreted in terms of cue-utilization strategies, which may adapt to target luminance when luminance remains invariant within trial blocks. Several alternative conceptions of the nature and locus of the cuevalidity effect in luminance detection are considered in light of these results.
\end{abstract}

Posner and his colleagues (e.g., Posner, Nissen, \& Ogden, 1978) have devised a simple, yet highly sensitive, procedure for investigating the effects of directed attention in visual target detection. The procedure consists of presenting a cue indicating the likely location of a signal that is to be detected, and then presenting the signal either at the cued location or (occasionally) at an uncued location. Detection latency under these conditions is shorter when the cue is valid than when it is invalid as a predictor of target location.

A complication of the basic procedure entails the addition of trials containing a noninformative (neutral) cue. Here, detection latency is often shorter in cued than in neutral trials, yielding what Posner terms an attentional benefit, and slower in uncued than in neutral trials, yielding an attentional cost. These effects hold even in the absence of eye movements (Posner, 1978). Posner has interpreted this pattern of results in terms of the assumptions that cuing directs the covert orientation of attention and that attention enhances the efficiency of sensory processes underlying detection.

Recently, Hughes (1984) used the Posner procedure to inquire into the location within the visual system of the processes underlying the cue-validity effect. One of the variables he investigated was target luminance, and he found (1) that reductions in target luminance produce overall increases in target-detection latency, but (2) that the magnitude of the cue validity effect (reaction time, RT, on invalid cue trials minus RT on valid cue trials)

Correspondence should be directed to H. L. Hawkins, Perceptual Sciences Program, Office of the Chief of Naval Research, Arlington, VA $22217-5000$. does not increase with reductions in target luminance. In fact, the cuing effect was reliably smaller for the lowest target luminance studied than for all others. The latter result was dismissed as the likely result of ceiling effects. Drawing on the logic of additive factors (Sternberg, 1969), Hughes interpreted the observed additivity across all other luminance conditions as showing that signal luminance and directed visual attention operate at separate stages in the signal detection process.

The Hughes result would seem to indicate that once visual attention is focused at a particular spatial location, the efficiency of processes leading to the detection of a target appearing at an alternative location (as on invalid cue trials) is unaffected by the salience of the signal appearing there. We found this puzzling. Intuitively, one might suppose that the speed with which attention can be oriented to an uncued location and engaged there (Posner, 1978) will increase with target salience. Even if one assumes that the detection of a luminance increment at an uncued location does not necessitate the reorientation of attention (e.g., LaBerge \& Brown, 1986), it might still be expected that the rate with which evidence of uncued target occurrence accrues within the sensory system will increase with target luminance. Seemingly compatible with these expectations are data (Julesz, 1981; LaBerge \& Brown, 1986) suggesting that the efficiency with which visual attention is utilized in detecting a target element or set of target elements appearing at uncued spatial locations will vary with the target's distinctiveness relative to background.

In the three experiments reported here we explored the possible basis of Hughes's findings. His conclusion that 
target luminance is additive with cue validity rests upon the assumption that his subjects responded to the precue in the same fashion under all luminance conditions; that is, that attention was directed to the cued position equally under all luminances. There is reason, however, for questioning this assumption. Target luminance was manipulated as a between-blocks variable. Target luminance was varied across trial blocks by means of neutral density filters, which were interposed between subjects and the entire stimulus display, including the locations of both targets and precues. The range of filters chosen by Hughes proved highly effective in manipulating detection latency. It may also have affected the ease with which subjects could extract useful information from the precue. More specifically, the dissociation of attention from the point of eye fixation on the basis of a central cue is an effortful process. Conceivably, this dissociation was made even more effortful by reductions in cue discriminability across luminance conditions in the Hughes study, reducing the likelihood that subjects actually used the cue to direct their attentional focus. This possibility is evaluated in Experiment 1 .

It is also conceivable that the blocked presentation of target luminances could have had a strategic effect of quite a different kind. Subjects may have assumed, correctly or not, that a full commitment of focal attention to the cued location under lower target-luminance conditions would lead to unacceptably high rates of detection failure on invalid cue trials. Accordingly, there may have been a greater tendency under low-luminance conditions to split attention between cued and uncued locations, or simply to hold attention at the fixation point, midway between target sites. The effect of either strategy would be to reduce the cue-validity effect. Thus, any differential cuing effect due to target luminance could have been offset by the subjects' attentional strategies. This possibility was tested in Experiments 2 and 3.

\section{EXPERIMENT 1}

In the first experiment, we varied target luminance across trial blocks by placing neutral density filters over target locations, but not over the location of the central precue. In this way, precue discriminability was held constant across target luminances.

\section{Method}

Stimulus display. A computer-controlled video raster monitor was used to generate the display, which consisted of a trial marker, a fixation cross, and a cuing arrow, all appearing at a central location, and a target that appeared either to the left or right of center. The display was viewed from a distance of $45.7 \mathrm{~cm}$. The fixation cross was $0.6^{\circ} \times 0.6^{\circ}$, the precue was a left- or right-oriented arrow subtending $0.6^{\circ} \times 0.9^{\circ}$, and the target subtended $0.05^{\circ}$. The targets appeared on a dark background $3.0^{\circ}$ center-to-center to either side of the cue. We varied target luminance by mounting neutral density filters over the two target positions. The three target luminances were 9.0, 3.0, and $1.0 \mathrm{mfL}$. For reasons indicated below, the experiment was conducted in a partially lighted room.
Task. The task was to respond to the occurrence of the target by depressing the space bar on a keyboard located on a table in front of the subject. Responses were made with the index finger of the preferred hand. Response latencies were measured by a hardware-based timer accurate in the microsecond range. The experimenter (K.R.) monitored eye movements on-line by means of a video camera, necessitating partial illumination of the experimental chamber. A preliminary study was carried out to assess the experimenter's ability to detect eye movements under the conditions of this experiment. The first author served as the subject in the preliminary study, which consisted of 264 trials. On a random onequarter of these trials, the subject made a $1.5^{\circ}$ eye movement, either to the left or right. Eighty-one percent of these eye movements were detected by the experimenter, and on no occasion did he report a movement when none was made. We reasoned that the experimenter's imperfect eye-movement detection would not constitute a problem for interpretation of experimental results unless both of the following conditions held: (1) the pattern of response latencies differed between trials on which eye movements were observed and those on which they were not; and (2) the frequency of observed eye movements differed across luminance blocks. Since performance patterns in the Posner cost-benefit procedure are not affected appreciably by the occurrence of eye movements (Shulman, Wilson, \& Sheehy, 1985), we anticipated no problem with this procedure.

Procedure. Following Posner et al. (1978), four types of trials were studied. These were designated valid, invalid, neutral, and catch trials. On valid trials, the target appeared on the side of fixation indicated by the cuing arrow. On invalid trials, the target appeared to the side opposite that indicated by the arrow. On neutral trials, a double arrow $(\rightarrow$ ) appeared, and the target occurred equally often to either side of fixation. On catch trials, no target appeared following cue presentation. There were $9.7 \%$ catch trials. Of the remainder, $76 \%$ were valid, $12 \%$ were invalid, and $12 \%$ were neutral. Each trial began with the presentation of the trial number, which was used by the experimenter as part of the eye-movement monitoring procedure. This remained on display for $1,500 \mathrm{msec}$; then it was replaced for $667 \mathrm{msec}$ by a fixation cross. The precue appeared $250 \mathrm{msec}$ following the offset of the fixation cross. The precue remained in view until the subject responded. The stimulus onset asynchrony between the precue and the $100-\mathrm{msec}$ target varied randomly between 750 and $1,500 \mathrm{msec}$. The interval between response and onset of the next trial was $1,000 \mathrm{msec}$.

Each subject participated in one practice session and three experimental sessions. The experimental sessions were conducted on separate days. Each experimental session consisted of three blocks of trials, one block at each target-luminance level. The order of luminance blocks was counterbalanced over subjects and experimental sessions. Subjects were informed about the predictive accuracy of the precue. They were instructed to maintain fixation throughout each trial. An error message appeared on the monitor when a subject committed an anticipation error, defined as a response on a catch trial or a response with a latency of less than $100 \mathrm{msec}$. Response latencies exceeding $1,200 \mathrm{msec}$ were defined as detection failures, and were signaled to subjects as they occurred by a "slow" message on the monitor.

Subjects. Six undergraduate students served as subjects. They were paid for their participation. All reported normal or correctedto-normal vision.

\section{Results and Discussion}

Anticipation errors and detection failures. Anticipation error rates were $2.1 \%$ on valid trials, $2.4 \%$ on invalid trials, $1.8 \%$ on neutral trials, and $3.0 \%$ on catch trials. An analysis of variance revealed no reliable differences in anticipation error rates across luminances, cue 
validity conditions, or days of practice. Overall, detection failure rates were very low: $0 \%$ on valid and neutral trials, and $0.2 \%$ on invalid trials. Latency data from error trials were excluded from further analysis.

Eye-movement data. Eye movements were observed on $1.8 \%, 2.1 \%$, and $2.0 \%$ of trials under high-, and medium-, and low-luminance conditions, respectively. An analysis of variance carried out on $X_{i}+1.0 \%$ transforms of these data revealed no reliable differences in eyemovement percentage across luminance or cue-validity conditions.

Reaction-time data. Table 1 shows mean correct RT for valid, neutral, and invalid cue trials under the three luminance conditions. Each cell in the table contains two values-mean RT including trials with recorded eye movements and (inparentheses) mean RT excluding trials with eye movements.

The question addressed in this experiment relates to the influence of target luminance on the magnitude of the cuevalidity effect-that is, on the difference in detection latency between trials containing valid cues and those containing cues that are not valid. More specifically, we asked whether the independence of cue-validity and targetluminance effects reported by Hughes (1984) could be attributed to luminance-dependent variations in cue discriminability. If so, the equalization of cue discriminability across target-luminance conditions in the present experiment should give rise to an interaction between target luminance and cue validity. To answer this question we carried out two separate three-factor repeatedmeasurements analyses of variance, one including data from trials with observed eye movements, the other excluding these trials. The three factors in these analyses were luminance (high, medium, low), cue validity (valid, invalid), and sessions $(1,2,3)$. The eye-movment inclusive analysis revealed significant luminance $[F(2,10)=$ 10.9] and cue validity effects $[F(1,5)=20.9$; both ps $<.001]$, and a significant sessions effect $[F(2,10)=$ $9.4, p<.01]$. Neither the luminance $\times$ cue validity interaction $(F<1.0)$ nor any other interaction approached significance. A virtually identical result appeared when data from eye-movement trials were excluded: luminance $[F(2,10)=10.0]$, cue validity $[F(1,5)=21.6$, $p<.001]$, and sessions $[F(2,10)=9.2, p<.01]$ were all significant, whereas the luminance $x$ cue validity interaction was not.

These results indicate that target luminance, at least across the range investigated here, is additive with the effects of cue validity. Thus, the present findings replicate those obtained by Hughes (1984) under his three highest target-luminance conditions, indicating that his results cannot be attributed to differences in precue discriminability across luminance blocks.

Given the results of the preliminary study described earlier in this report, we assume the experimenter detected a major portion of the eye movements that occurred during this experiment. On this assumption, the present results indicate that eye movements have a negligible impact on the pattern of response latencies obtained in tasks such as the present one, which impose no acuity demand on subjects. A similar conclusion was reached by Shulman et al. (1985).

\section{EXPERIMENT 2}

The second experiment provides a test of the idea that subjects in the Hughes (1984) experiment strategically varied their cue-utilization strategies across blockedluminance conditions. To eliminate possible strategic differences across luminance conditions, target luminance was manipulated as a within-blocks variable.

\section{Method}

Stimuli and Procedure. The stimulus display differed from that in Experiment 1 only with respect to target characteristics. Targets were created from rectangular graphics characters with variable pixel density, combined with predefined brightness codes (white and light gray), in Turbo Pascal. The highest luminance target consisted of Turbo Pascal character No. 219 with the color code white. The intermediate luminance target consisted of No. 177 (white), and the lowest luminance target consisted of No. 176 (light gray). Each target subtended a visual angle of $0.125^{\circ}$ horizontally and $0.25^{\circ}$ vertically. A neutral density filter was placed over each of the two target locations, yielding luminances of $4.0, .80$, and $.15 \mathrm{mfL}$ for the high-, intermediate-, and low-luminance targets, respectively. The filters were added for two reasons. First, we wished to shift target intensities into a range where strong-luminance main effects would be obtained (cf. Hughes, 1984). Second, we wished to minimize the possibility that subjects might perceive textural differences among the graphics characters used in the experiment. Preliminary observations indicated that the use of high-density filters, coupled with brief $(100 \mathrm{msec})$ target duration, effectively eliminates perceived textural differences among the characters used.

The task was identical in all respects to that in Experiment 1. The only procedural changes were that target luminance varied randomly within trial blocks and eye movements were not monitored. The decision to eliminate monitoring of eye movements seemed justified because the results of the first experiment revealed (1) no effect of target luminance or cue validity on eye-movement frequency,

Table 1

Mean Correct Reaction Time (in msec) under Valid, Neutral, and Invalid Cuing Conditions as a Function of Target Luminance

\begin{tabular}{|c|c|c|c|c|}
\hline \multirow{2}{*}{$\begin{array}{c}\text { Target } \\
\text { Luminance }\end{array}$} & \multicolumn{3}{|c|}{ Cue Validity Condition } & \multirow{2}{*}{$\begin{array}{l}\text { Invalid Cue RT } \\
\text {-Valid Cue RT }\end{array}$} \\
\hline & Valid & Neutral & Invalid & \\
\hline High & $267(265)$ & $273(270)$ & $305(304)$ & $38(39)$ \\
\hline Medium & $265(265)$ & 274 (272) & $303(302)$ & $38(37)$ \\
\hline Low & $300(298)$ & $305(304)$ & $333(330)$ & $33(32)$ \\
\hline
\end{tabular}

Note-The right-hand column gives the reaction-time differences between invalid and valid cuing conditions. The values in parentheses exclude data from trials with observed eye movements; the remaining values include data from these trials. 


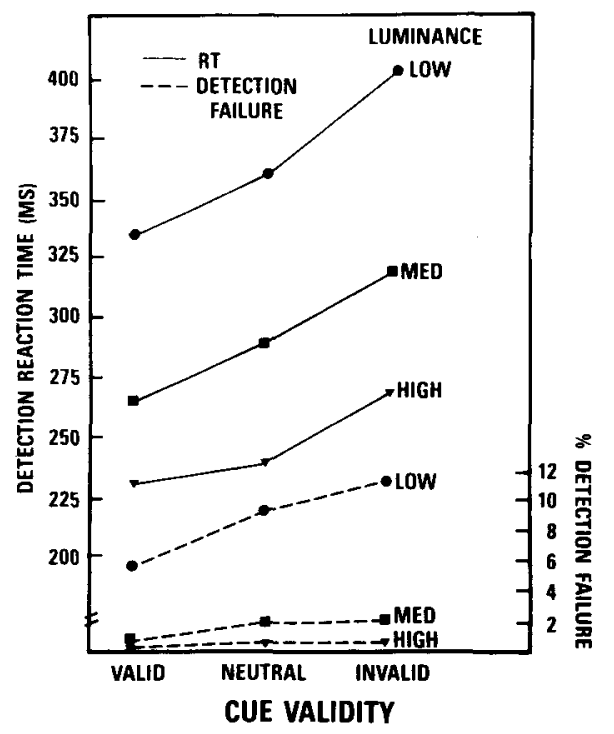

Figure 1. Mean correct reaction time and percent detection failures under valid, neutral, and invalid cuing conditions as a function of target luminance.

and (2) that eye movements had no appreciable effect on latency patterns across target-luminance and cue-validity conditions. Furthermore, since target luminance varied randomly within trial blocks in the present experiment, subjects should not have been able to vary the frequency of their anticipatory eye movements across target luminances on any systematic basis.

Subjects participated in one practice session and three experimental sessions, each consisting of three trial blocks. Blocks were identical, with the exception that the order of trial types was separately randomized for each block.

Subjects. Seven students, all reporting normal or corrected vision, served as subjects. They were compensated as in Experiment 1.

\section{Results and Discussion}

Anticipation errors. Anticipation error rates were $3.2 \%$ on valid trials, $3.4 \%$ on invalid trials, $2.6 \%$ on neutral trials, and $3.4 \%$ on catch trials. An analysis of variance indicated no reliable differences in anticipation-error rates across intensities, cue validity, or sessions.

Detection failures. Detection-failure rates are shown in Figure 1. An analysis of variance carried out on $X_{i}+1.0 \%$ transforms of these data revealed that luminance $[F(2,12)=3.88]$, cue validity $[F(2,12)=7.57]$, sessions $[F(2,12)=3.34]$, and the luminance $\times$ cue validity in- teraction $[F(4,24)=2.91]$ were all significant, with $p<.05$. The sessions main effect indicates a reduction with practice in the detection-failure rate.

Reaction-time data. Mean correct RT for valid, neutral, and invalid cue trials under each of the three luminance conditions is depicted in Figure 1 and Table 2. The question we sought to answer in this experiment concerns the additivity of target luminance and cue validity observed by Hughes (1984) when luminance remained invariant within trial blocks. Would the additivity relation remain in effect when luminances were mixed within trial blocks? To answer this question we carried out a repeated measures analysis of variance with luminance (high, medium, low), cue validity (valid, invalid), and sessions $(1,2,3)$ as variables. The results revealed that luminance $[F(2,12)=118.5]$, cue validity $[F(1,6)=56.0]$, and the luminance $\times$ cue validity interaction $[F(2,12)=15.9]$ were all significant $(p<.001)$. All 7 subjects showed a cue-validity effect that was greater in absolute magnitude under low than under high luminance.

These results are consistent with the idea that the additivity of target luminance and cue validity obtained by Hughes (1984), and replicated in our first experiment, is a consequence of systematic variations across blocked target luminances in the extent of the attentional focus induced by precuing. Subjects may be less inclined to commit attention exclusively or fully to the cued location during low, relative to high, luminance blocks. When this strategic option is removed by mixing luminances within trial blocks, as in Experiment 2, luminance and cuevalidity effects become overadditive. The pattern of detection failures appearing in Experiment 2 seems another likely consequence of the procedurally induced invariance of attentional strategy across target luminances. Indeed, any given cue-utilization strategy, with the exception of the most conservative (least cue-committed), might be expected to produce a difference in detection-failure rate between cued and uncued trials, which would increase with reductions in target luminance.

One objection that could be raised with respect to this analysis is that Experiments 1 and 2 differed not only in blocking procedure; they also differed in the specific method by which luminance was manipulated and in the strength of this manipulation. Since our arguments are based on the assumption that the blocking procedure was the critical factor that differentiated the results of Experiments 1 and 2, these confounds were eliminated in Experiment 3.

Table 2

Mean Correct Reaction Time (in msec) under Valid, Neutral, and Invalid Cuing Conditions as a Function of Target Luminance (Experiment 2)

\begin{tabular}{|c|c|c|c|c|}
\hline \multirow{2}{*}{$\begin{array}{c}\text { Target } \\
\text { Luminance }\end{array}$} & \multicolumn{3}{|c|}{ Cue Validity Condition } & \multirow{2}{*}{$\begin{array}{l}\text { Invalid Cue RT } \\
\text { - Valid Cue RT }\end{array}$} \\
\hline & Valid & Neutral & Invalid & \\
\hline High & 232 & 241 & 272 & 40 \\
\hline Medium & 267 & 285 & 320 & 53 \\
\hline Low & 335 & 359 & 402 & 67 \\
\hline
\end{tabular}

Note-The right-hand column gives the reaction-time differences between invalid and valid cuing conditions. 


\section{EXPERIMENT 3}

\section{Method}

Stimuli and Procedure. The stimulus display, target-luminance manipulation, specific target luminances tested, and task were all identical to those of Experiment 2 . The only procedural difference was that target luminance varied across rather than within trial blocks. The order of luminance blocks was counterbalanced over subjects and experimental sessions.

Subjects. Nine students and three clerical staff, all reporting normal or corrected vision, served as subjects. They were compensated as in the first two experiments.

\section{Results and Discussion}

Anticipation errors and detection failures. Anticipation error rates were $1.4 \%$ on valid trials, $2.2 \%$ on invalid trials, $2.0 \%$ on neutral trials, and $2.3 \%$ on catch trials. Detection failure rates were $0.2 \%$ on valid trials, $0.4 \%$ on neutral trials, and $1.1 \%$ on invalid trials. Analyses of variance carried out separately on anticipation error and detection failures indicated no reliable differences across luminance blocks, cue validity conditions, or days of practice.

Reaction time data. Table 3 shows mean correct RT for valid, neutral, and invalid cuing under the three target luminances. Data from valid and invalid cuing conditions were subjected to a repeated measures analysis of variance with target luminance (high, medium, low), cue validity (valid, invalid), and sessions $(1,2,3)$ as factors. The results revealed that luminance $[F(2,22)=111.0]$ and cue validity $[F(1,11)=93.1]$ were significant $(p<.001)$. Although the absolute magnitude of the cuevalidity effect tended to decline with decreasing target luminance, the luminance $\times$ cue validity interaction did not approach significance.

Target luminance varied across trial blocks in the present experiment and within trial blocks in Experiment 2. The experimental procedures of these two experiments differed in no other respects. We may thus conclude that the overadditivity of luminance and cue validity observed in Experiment 2 was due to the mixing of luminances within trial blocks. Accordingly, the present results lend support for our suggestion that the blocking of target luminance promotes strategic variations in the degree to which attention is committed to the cued location. Of course, our argument would have been strengthened further had we found that the two variables were underadditive under blocked conditions. However, as the reader will recall, Hughes (1984) obtained just this result for his lowest luminance condition relative to all others. Measured luminance in this condition was lower than in our low-luminance condition, and overall RT was higher. Hughes interpreted the underadditivity as a ceiling effect. However, it is unclear what this ceiling effect might be. Given the results reported here, the more plausible interpretation is that the blocking of target luminance encourages adaptive variations in the cue-based commitment of visual-spatial attention.

\section{GENERAL DISCUSSION}

The results of the experiments reported here suggest two noteworthy implications, one methodological and one theoretical. First, when considered in light of Hughes (1984) and our Experiments 1 and 3, the results of Experiment 2 indicate that the allocation of attention in spatial cuing is not determined exclusively by cue validity. If subjects believe, correctly or not, that the full, cue-based allocation of attention will result in unacceptably high detection-failure rates for targets at an uncued location, they may be disinclined to commit attention fully or exclusively to the cued location. When blocked experimental conditions, such as target luminance, differ with respect to the likelihood of detection failure, subjects appear to reallocate their attention accordingly. If such conditiondependent strategies are not taken into consideration as experimental factors, they can lead to the misinterpretation of experimental results. A related effect, with rather different design implications, has been reported by Posner (1978). The reference study (Posner et al., 1978) investigated the effects of target eccentricity in the spatial cuing paradigm. When target eccentricity was treated as a blocked variable, no differences in the size of the cuevalidity effect were obtained between parafoveal and more eccentric targets. When eccentricities were mixed within trial blocks, however, more peripheral targets showed larger cue-validity effects than did parafoveal targets. Posner interpreted this surprising result to mean that subjects biased their attention toward peripheral target locations under the mixed condition, mistakenly believing that the peripheral target was more critical, or perhaps more subject to detection failure. Following this interpretation, it was the mixed-eccentricity condition that produced the misleading outcome, and not the blocked condition. The point to be emphasized is that strategic factors can in-

Table 3

Mean Correct Reaction Time (in msec) under Valid, Neutral, and Invalid Cuing Conditions as a Function of Target Luminance (Experiment 3)

\begin{tabular}{|c|c|c|c|c|}
\hline \multirow{2}{*}{$\begin{array}{c}\text { Target } \\
\text { Luminance }\end{array}$} & \multicolumn{3}{|c|}{ Cue Validity Condition } & \multirow{2}{*}{$\begin{array}{l}\text { Invalid Cue RT } \\
\text {-Valid Cue RT }\end{array}$} \\
\hline & Valid & Neutral & Invalid & \\
\hline High & 275 & 293 & 322 & 47 \\
\hline Medium & 302 & 310 & 347 & 45 \\
\hline Low & 354 & 364 & 396 & 42 \\
\hline
\end{tabular}

Note-The right-hand column gives the reaction-time differences between invalid and valid cuing conditions. 
fluence outcomes in spatial cuing, and must be taken into consideration when interpreting experimental results.

A second implication of the present results relates to Hughes's (1984) conclusion that the effects of target luminance and directed attention operate at separate stages of the signal-detection process. We have argued that the additivity (more correctly, the underadditivity) of these effects in the Hughes experiment resulted from strategic factors operating across blocked luminance conditions. When the strategic option is removed by mixing target luminances within blocks, luminance and cue-validity effects become overadditive.

How is this overadditivity to be interpreted? In terms of the logic of additive factors (Sternberg, 1969), our results imply that target-luminance and cue-validity effects operate at a common stage in the sequence of events leading up to the detection response. This is not to imply that the two variables initiate their influence at the same point. Luminance effects start at photoreception (Baylor \& Hodgkin, 1973), whereas the effects of spatial cuing surely are initiated at a later stage of processing. It appears that the effects of target luminance perseverate through the visual system at least up to the stage where the effects of spatial cuing become operative. It is at this later stage that the two effects are colocalized.

At what processing stage are cue-validity and targetluminance effects colocalized, and by what mechanism do they interact? Posner (1978) has interpreted the cuevalidity effect on the assumption that cue-oriented attention enhances the efficiency of processing within the sensory pathways leading to detection. Others (e.g., Duncan, 1981; Müller \& Findlay, 1987; Shaw, 1978, 1984) disagree, arguing that cue-validity effects in luminance detection are more likely a consequence of decision bias than of sensory facilitation. Cue-validity effects are observed under conditions in which targets are more likely to occur at cued than at uncued locations. Consequently, subjects may simply require less sensory evidence, and therefore less stimulus sampling time, in order to decide that a target has appeared at a cued location. Evidence favoring the decision-bias account appears in studies by Shaw (1984) and Müller and Findlay. Shaw found that the magnitude of the luminance-detection decrements obtained when attention is divided rather than focused is consistent with the assumption that spatial cuing induces response bias; but it is well below what might be expected on the assumption that spatial cuing also facilitates sensory processing. Consistent with this, Müller and Findlay found that luminance-detection performance for targets at cued versus uncued locations differs with respect to criterion setting (beta) but not with respect to measured sensitivity.

However, two lines of evidence raise questions regarding the adequacy of the decision-bias interpretation. One of these comes from a study by Shulman, Sheehy, and Wilson (1986). The task was to detect a target that could appear at any of five horizontally arrayed locations to one side of fixation. One of the five locations was cued on each trial. For trials containing a target, the target probability was .76 for the cued location and .06 for each of the remaining four locations. The detection latency was found to vary as a joint function of retinal eccentricity and the distance between cued and target locations. This result can be readily accommodated by the idea that attention forms a gradient of sensory facilitation around the cued location (Shulman, Sheehy, \& Wilson, 1986), or perhaps by the idea that luminance detection requires attention to be reoriented from the cued to the target location through a process whose duration depends on the spatial distance traversed (but see below). However, since uncued locations had equal probabilities of target occurrence in this study, it is unclear how the results could be accommodated by the decision-bias account. To explain these results, the decision-bias account would have to be modified so that the decision criterion for a given location would depend not only on the probability that it contained a target, but also on its spatial distance from the cued location-an assumption that does not follow directly from the theoretical signal-detection account. Moreover, although it is plausible that subjects could maintain distinct decision criteria for two separate spatial locations, it would be incredible for them to maintain distinct criteria for five.

The second line of evidence posing difficulties for the decision-bias account appears in a series of event-related potential (ERP) studies reported in Mangun (1987) and Mangun, Hansen, and Hillyard (1987). Subjects were cued to attend to a location in either the left or right visual field, and were then presented stimuli at either the cued or uncued location. ERPs over occipital regions had a higher amplitude when elicited by targets at cued locations than when elicited by targets at uncued locations. Moreover, these enhancements occurred in ERP components that appeared as early as $90-100 \mathrm{msec}$ following the target's onset, and peaked over prestriate cortical areas of the occipital lobe. These results strongly suggest that visual-spatial attention modulates sensory processing within the visual system, and that such modulatory effects can operate during luminance detection. ${ }^{1}$

The Shulman et al. (1986) and Mangun et al. (1987) findings suggested to us the following interpretation of Shaw (1984) and Müller and Findlay (1987), and of the results described in the present report: Assume that cuing orients attention and that the attentional mechanism generates the excitatory activation of structures within the sensory pathway(s) coding inputs from the cued location. (Alternatively, it might be assumed that attentional orientation creates the inhibition of structures coding inputs from ignored spatial locations, or produces a combination of excitation and inhibition. But this does not matter for what follows. For simplicity's sake, we will assume that the modulatory effect of focused attention is strictly excitatory.) In terms of signal-detection theory, the sensory states of affairs at cued and uncued locations can then be represented as follows: In structures coding input from a cued location, the distribution of sensory strengths ex- 
perienced on trials that contain a brief luminance increment is a function of neural noise, signal strength, and an attentional activation parameter, $a$. The strength distribution for trials on which no signal occurs is a function of neural noise and the parameter $a$. At an uncued location, the two strength distributions are a function, respectively, of signal (S) plus noise, $(\mathrm{N})$, and of noise alone. In this proposal, the $\mathrm{SN}$ and $\mathrm{N}$ distributions at the cued location are both shifted additively up the continuum of sensory strength by a quantity, $a$, relative to those generated at the uncued location. Now suppose a single decision criterion, defined at a given point along the strength continuum, is applied to the analysis of activity at both cued and uncued locations. The resulting system would produce an increased probability of both hits and false alarms for the cued, relative to uncued, location. This would produce differences in measured "criterion setting' but not in measured sensitivity. That is, it would yield the pattern of luminance detection outcomes reported by Müller and Findlay (1987) and Shaw (1984), even though the effects of attentional modulation are postulated to occur strictly within the sensory system. ${ }^{2,3}$

This analysis can readily be extended to account for major features of the data reported here. As pictured in Figure 2, we assume that target luminance determines the latency and the rate at which excitation builds in sensory structures underlying detection (cf. Nissen, 1977). We assume further that once attention is oriented towards a cued location, the level of activity within structures coding input at this location is increased by a constant quantity, $a$, relative to that at uncued locations. Assuming that a common criterion exists across target luminances and across cued and uncued locations in Experiment 2, the difference in time to criterion between cued and uncued target locations will be greater for low than for high luminance targets. In fact, this difference will be directly proportional to the inverse of the difference, across luminances, in slope of the evidence-acquisition function. To account for the pattern of detection failures appearing in Experiment 2 (see Figure 1), we assume that the probability that the activity generated by the $100 \mathrm{msec}$ target used here will achieve criterion declines as the target luminance is reduced.

We have suggested that under blocked luminance conditions, as in Hughes (1984) and in the present Experiments 1 and 3, subjects may alter their strategies for cue utilization across trial blocks, reducing the extent of their attentional commitment to the cued location during blocks containing low-luminance targets. Following the account developed here, this would mean that the difference in activation level between cued and uncued locations-that is, the value of the attention parameter $a$-would tend to

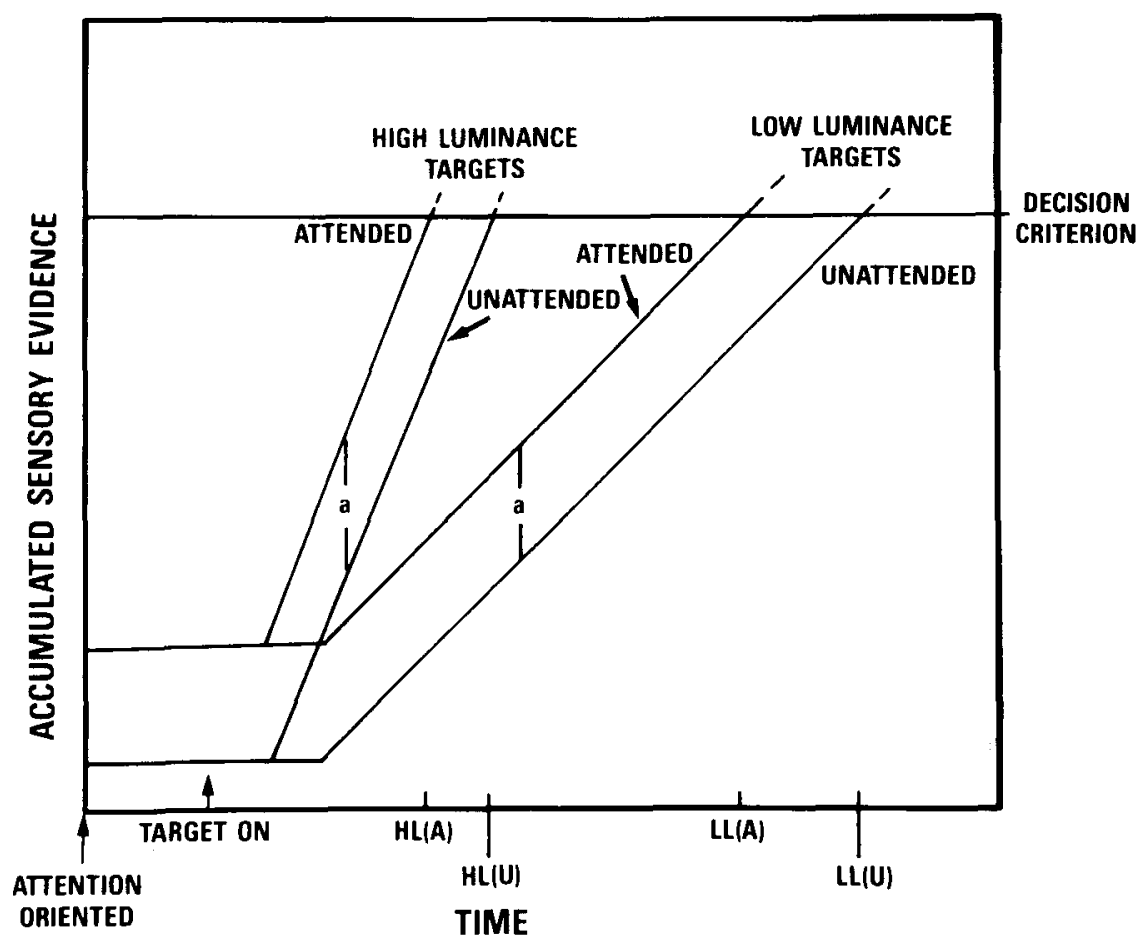

Figure 2. Hypothetical evidence-acquisition curves for high- versus low-luminance targets at attended and unattended spatial locations. HL(A) indicates the hypothetical point in time at which evidence generated by a high-luminance target at the attended location achieves criterion. For simplicity, the evidence-acquisition functions are depicted as linear with time. This is not necessary for the proposed account. The only requirement is that evidence accrues as a monotonic function of time, and that the differential effect of $a$ across target luminances is preserved in these functions. 
decline as target luminance is reduced. Consequently, the overadditivitiy of cue validity and target luminance obtained under mixed-luminance blocks will decline or perhaps even reverse under blocked luminance conditions, as in Hughes (1984).

In the account proposed here, the cue-validity effect in luminance detection is wholly attributable to attentionally mediated differences in the activation of sensory structures coding cued versus uncued target locations. This view is similar to that of Posner (1978), in assuming that the cue-validity effect has a sensory locus. But our account differs from that of Posner in at least one notable respect. For example, Posner, Walker, Friedrich, and Rafal (1984) have assumed that not only does attention facilitate processing through sensory input pathways, but it normally also must be oriented towards the target location before detection can occur. Thus, when a target appears at an uncued location, subjects must disengage their attention from the cued location, move it to the target's actual location, and then engage it in order to facilitate sensory processing. Posner assumes that these three timeextended operations-disengagement, movement, and engagement-contribute to the latency difference observed between targets at cued and uncued locations. This view can accommodate the results of the second experiment reported here on the basis of two assumptions: first, that the disengagement operation will not be initiated on invalid cue trials until evidence of target occurrence at the uncued location has accumulated to some necessary (criterial) level; and second, that the accrual rates for evidence are slower with respect to low-luminance targets, and that therefore more time will elapse after the onset of a low-luminance target before sufficient evidence has accrued to initiate attentional disengagement.

In contrast, the account developed here assumes that the cue-validity effect in luminance detection is due entirely to attentionally mediated differences in activity level between sensory structures coding inputs at cued versus uncued spatial locations. In particular, we assume that oriented attention facilitates the speed of luminance detection, but that it is not required in order to achieve it. We know of no adequate basis in the literature for choosing between these two accounts. Although the presence of costs and benefits might be used to argue the necessity of oriented attention in luminance detection, this is not persuasive. Under neutral cuing, subjects may distribute their attention between target sites, with some efficiency loss relative to more focally attended sites and some gain relative to unattended sites, or they may orient attention sometimes to one location and at other times to the other. On trials containing an invalid cue, either tactic would produce costs and benefits in the absence of attentional reorientation. Our preference for the view developed here is based on two nonempirical considerations. First, it is simpler. Second, according to Posner, the disengagement of attention from a cued location normally is precipitated by the detection of sensory activity at an uncued location.
If this detection of activity at an uncued location can elicit the disengagement operation, as postulated by Posner, why should it not enable a detection response as well? What is the necessity of attentional reorientation in luminance detection?

\section{REFERENCES}

Baylor, D. A., Hodgkin, A. L. (1973). Detection and resolution of visual stimuli by turtle photoreceptors. Journal of Physiology. 234, 163-198.

Duncan, J. (1981). Directing attention in the visual field. Perception \& Psychophysics, 30, 90-93.

HuGHES, H. C. (1984). Effects of flash luminance and positional expectancies on visual response latency. Perception \& Psychophysics, 36, 177-184.

JuLESZ, B. (1981). Textons, the elements of texture perception, and their interactions. Nature, 290, 91-97.

LABERGE, D., \& Brown, V. (1986). Variations in size of the visual field in which targets are presented: An attentional range effect. Perception \& Psychophysics, 40, 188-190.

MANGUN, G. R. R. (1987). Mechanisms of visual selective attention: Analyses with event-related brain potentials in humans. Unpublished doctoral dissertation, University of California, San Diego.

Mangun, G. R. R., Hansen, J. C., \& Hillyard, S. A. (1987). The spatial orienting of attention: Sensory facilitation or response bias? In R. Johnson, J. W. Rohrbaugh, \& R. Parasuraman (Eds.), Current trends in event-related potential research (EEG Supplement 40; pp. 118-124). Amsterdam: Elsevier.

Müller, H. J., \& Findlay, J. M. (1987). Sensitivity and criterion effects in the spatial cuing of visual attention. Perception \& Psychophysics, 42, 383-399.

NisSEN, M. J. (1977). Stimulus intensity and information processing. Perception \& Psychophysics, 22, 338-354.

POSNER, M. I. (1978). Chronometric explorations of mind. Hillsdale, NJ: Erlbaum.

Posner, M. I., Nissen, M. J., \& Ogden, W. C. (1978). Attended and unattended processing modes: The role of set for spatial location. In H. L. Pick \& I. J. Saltzman (Eds.), Modes of perceiving and processing information (pp. 117-140), Hillsdale, NJ: Erlbaum.

Posner, M. I., Walker, J. A., Friedrich, F. J., Rafal, R. D. (1984). Effects of parietal injury on covert orienting of attention. Journal of Neuroscience, 4, 1863-1874.

SHAW, M. L. (1978). A capacity allocation model for reaction time. Journal of Experimental Psychology: Human Perception \& Performance, 4, 586-598.

SHAW, M. L. (1984). Division of attention among spatial locations: A fundamental difference between the detection of letters and detection of luminance increments. In H. Bouma \& D. G. Bouwhuis (Eds.), Attention and performance $X$ (pp. 189-206). Hillsdale, NJ: Erlbaum.

Shulman, G. L., Wilson, J., Sheehy, J. B. (1985). Spatial determinants of the distribution of attention. Perception \& Psychophysics, 37, 59-65.

Shulman, G. L., Sheehy, J. B., \& Wilson, J. (1986). Gradients of spatial attention. Acta Psychologica, 61, 167-181.

STERNBERG, S. (1969). The discovery of processing stages: Extensions of Donders' method. In W. G. Koster (Ed.), Attention and Performance II (pp. 276-315). Amsterdam: North-Holland.

\section{NOTES}

1. It would be inappropriate to argue from the results of Mangun et al. (1987) and Shulman et al. (1987) that the cue-validity effect is entirely a product of sensory factors. The more appropriate conclusion is that the effect is due, at least in significant part, to such factors. Whether or not decision-level factors are also involved and, if so, what the relative contributions of sensory- and decision-level factors are, remain unresolved issues. 
2. Müller and Findlay (1987) also investigated conditions in which the task was to determine the presence or absence of a specified target that could appear at either a cued or an uncued spatial location. In this recognition task, performance for targets at cued and uncued locations differed with respect to both decision criterion and sensitivity. In a related vein, Shaw (1984) reported declines in target-recognition performance between focused-and divided-attention conditions-declines that were substantially greater than what might be expected on the basis of decision bias alone. Thus, the state of affairs in target recognition clearly differs from that in luminance detection. The account of spatial cuing developed here is not compromised by this finding, but does require extension in order to account for it. A detailed treatment of cuing effects in target recognition extends beyond the scope of the present report. In brief, however, we suggest the following possibility: Suppose that a subject must judge whether a spatially arrayed set of stimuli contains a prespecified target. Suppose that targets and distractors are not perfectly discriminable: that is, for instance, that targets are defined by the feature set, $f 1, f 2$, and $\mathrm{f}$, and distractors, by the (overlapping) set, $f 2, f 3$, and $f 4$. Assume there exists for each target location a neural structure (target representation) that accumulates activation from a set of sensory pathways, each member of which is tuned to one of the target's defining features, and that a recognition response occurs if the strength of activation in one or more of these accumulators exceeds a specified criterion value. Since targets and distractors contain common features, assume that their strength distributions overlap within target structures. Now assume that the function of focused attention is to contribute top- down excitation to the set of sensory pathways (coding $f 1, f 2, f 3$ ) that feed the target structure at the cued location. Accordingly, the effect of attention is to upshift both target- and distractor-strength distributions at the cued, as opposed to uncued, locations. However, the upshift will be greater for the target distribution than for the distractor distribution because attention activates the $\mathrm{f} 1$ pathway, which is unique to the target, as well as the $\mathrm{f} 2$ and $\mathrm{B}$ pathways commonly activated by targets and distractors. Assuming a common criterion placement along the strength continuum for inputs at cued and uncued locations (as in our account of the luminance-detection data), this conception provides an explanation of the Müller and Findlay (1987) and Shaw (1984) results. The attentionally mediated upshift of strength distributions for both targets and distractors at the cued location will produce a measured decision bias, and the relatively greater upshift for the target distribution will produce an increase in measured sensitivity.

3. If one assumes a broad attentional gradient (Shulman et al., 1986), $a$ would be a function of the difference between cued and uncued locations in gradient height. On the assumption of a sufficiently narrow gradient, $a$ would be a function of the gradient height at the cued location. The general logic of the account is preserved across these alternatives. Moreover, a similar interpretation would follow if the effect of $a$ were multiplicative rather than additive.

(Manuscript received July 17, 1987; revision accepted for publication May 9, 1988.) 